\title{
Médiévales
}

Langues, Textes, Histoire

46 | printemps 2004

Éthique et pratiques médicales

\section{Éthique et pratiques médicales aux derniers siècles du Moyen Âge}

\section{Marilyn Nicoud}

\section{(2) OpenEdition}

1 Journals

\section{Édition électronique}

URL : https://journals.openedition.org/medievales/1147

DOI : $10.4000 /$ medievales. 1147

ISSN : $1777-5892$

\section{Éditeur}

Presses universitaires de Vincennes

\section{Édition imprimée}

Date de publication : 1 juin 2004

Pagination : 5-10

ISBN : 2-84292-151-8

ISSN : 0751-2708

\section{Référence électronique}

Marilyn Nicoud, «Éthique et pratiques médicales aux derniers siècles du Moyen Âge », Médiévales [En ligne], 46 I printemps 2004, mis en ligne le 13 mars 2006, consulté le 22 avril 2022. URL : http:// journals.openedition.org/medievales/1147 ; DOI : https://doi.org/10.4000/medievales. 1147

Ce document a été généré automatiquement le 22 avril 2022

Tous droits réservés 


\title{
Éthique et pratiques médicales aux derniers siècles du Moyen Âge*
}

\author{
Marilyn Nicoud
}

1 Médicalisation, tel est le terme souvent employé pour qualifier les évolutions que connaît la pratique médicale à partir du XIII ${ }^{\mathrm{e}}$ siècle, encore que celles-ci soient limitées, à cette date, aux pays méditerranéens. Ce vocable défini par Joseph Shatzmiller et Michael McVaugh ${ }^{1}$ désigne une certaine institutionnalisation et professionnalisation de la médecine, dans ses aspects à la fois théoriques et pratiques. Création de facultés, constitution d'un curriculum d'études, contrôle de l'accès à la profession par des instances $a d$ hoc, formations de collèges de praticiens, appointements par les communes de médecins salariés illustrent les multiples facettes des transformations qui s'opèrent, relayées par des autorités publiques soucieuses, au nom d'un principe d'utilité commune, de disposer d'un corps médical apte à prendre en charge la santé des populations. Dans le droit romain retrouvé, le médecin puise sa justification sociale certes, mais également éthique ${ }^{2}$.

2 Si les travaux passés ont déjà éclairé nombre de ces aspects - que l'on songe aux études portant sur l'enseignement universitaire ${ }^{3}$, la diversité du milieu médical ${ }^{4}$ et les difficultés d'en exclure les femmes ${ }^{5}$, les charlatans et autres empiriques, sur les praticiens communaux des villes ${ }^{6}$, mais aussi sur les médecins ruraux ${ }^{7}$, voire sur les politiques de prévention face aux épidémies ${ }^{8}$, sans oublier la transformation des hôpitaux d'établissements charitables en établissements de soins ${ }^{9}-$, une autre dimension de cette médicalisation médiévale a peut-être été plus négligée: ce qui touche notamment aux relations qu'entretiennent patients et médecins et qui conduit souvent les praticiens à mieux définir non seulement l'objet même de leur art, le champ de leurs compétences, l'éthique à suivre dans la pratique, mais aussi les rapports que leur discipline doit entretenir avec d'autres savoirs proches. C'est ce vaste domaine que le présent volume a pour ambition d'aborder.

3 À l'image de certaines attitudes actuelles, ces rapports entre patients et praticiens paraissent complexes, difficiles, voire ambigus ${ }^{10}$. Si les médias se font l'écho des débats éthiques de la médecine contemporaine - qu'ils portent sur la responsabilité médicale, 
sur le problème de la fin de vie et de sa médicalisation, ou sur des questions en apparence plus futiles, telle la demande accrue en matière de chirurgie esthétique -, les sources médiévales, qu'elles proviennent des traités savants ou des archives, révèlent des enjeux similaires et font entendre l'écho lointain de ces débats. En effet, au prisme déformant de l'actualité, ces questions nous paraissent contemporaines, alors même qu'elles agitaient déjà le monde antique comme le souligne Danielle Gourévitch dans le Triangle hippocratique ${ }^{11}$. Cherchant à analyser les représentations sociales et individuelles de la maladie, elle aborde la difficile question de la communication entre patient et médecin et les problèmes liés à la déontologie médicale; plus récemment, ces questions relatives à l'émergence dans l'Antiquité grecque et romaine d'une éthique ou d'une philosophie médicale, mettant notamment l'accent sur la relation médecinpatient, ont fait l'objet des très intéressantes communications publiées par la Fondation $\operatorname{Hardt}^{12}$.

4 Au Moyen Âge, c'est sans doute dans les sphères des élites que ces rapports entre malades et praticiens peuvent être le mieux éclairés. La documentation nous montre ainsi la précoce émergence de la figure du médecin de cour, appointé par les grands, au chevet de ses patients, présent au gré de leurs déplacements, et qui tente souvent de concilier cette pratique astreignante et un enseignement professionnel. La cour pontificale au xIII ${ }^{\mathrm{e}}$ siècle ${ }^{13}$, la couronne d'Aragon dans la seconde moitié du siècle ${ }^{14}$ témoignent de l'ancienneté d'un phénomène que l'on voit se poursuivre dans les cours princières de l'Italie centrale et septentrionale. $\mathrm{Au} \mathrm{Xv}^{\mathrm{e}}$ siècle, dans la Milan des Sforza, par exemple, les échanges épistolaires soulignent la place qu'occupent santé et maladie dans les relations sociales, et rendent plus généralement compte d'un très présent souci de soi, de son bien-être et de sa santé ${ }^{15}$. Mais ce souci est tributaire du rapport non univoque qui se crée entre le professionnel et son patient: si les manuels de déontologie insistent sur la nécessaire obéissance du patient et sur la relation de confiance que doit savoir susciter le praticien ${ }^{16}$, la réalité montre souvent qu'entre les recommandations de ce dernier et l'attitude du premier existe un décalage bien difficile à combler: si le médecin apparaît bien comme une figure d'autorité, c'est aussi un homme de contrainte ; ses prescriptions et ses conseils peuvent en effet être rejetés par le patient, surtout lorsque s'opposent aux interprétations médicales de la maladie les représentations que s'en fait le malade et son propre vécu, qui constitue d'ailleurs - et ce n'est là qu'une contradiction supplémentaire - l'un des éléments sur lesquels le praticien s'appuie pour établir son diagnostic. Comment interpréter par exemple les rébellions de Francesco Sforza contre son médecin qu'il soumet à la même diète que celle que ce dernier lui avait imposée pour qu'à son tour, il en éprouve les désagréments ${ }^{17}$ ? Que dire de prescriptions préventives ou conservatoires ${ }^{18}$ imposées à ceux qui se sentent bien portants? Quel poids accorder à la parole médicale, quelle portée pratique lui consentir? Comment enfin mettre en relation ce que nous disent les discours des médecins et ce qu'en font les patients?

5 Ces problèmes ont partie liée avec le statut social du médecin et avec les conditions mêmes dans lesquelles il exerce son métier. Si la professionnalisation du métier médical, progressive à partir du XIII ${ }^{\mathrm{e}}$ siècle, a tendu à exclure de son exercice tous ceux qui ne disposaient pas des titres universitaires requis, la prolifération des vocables employés pour désigner ce tissu social - médecins, physici, chirurgiens, barbiers, mires, empirici, matrones... ${ }^{19}$ - en montre assez l'hétérogénéité et la difficile fermeture de la profession aux seuls autorisés. D'évidence, selon la réputation du médecin, en vertu de 
la qualité du patient, les relations varient, non seulement entre malade et praticien, mais aussi entre professionnels, lorsqu'ils sont plusieurs au chevet d'un même client.

Aussi certains auteurs n'hésitent-ils pas à conseiller le recours à la ruse pour obtenir cette confiance du patient à l'image, au XII ${ }^{\mathrm{e}}$ siècle, de l'œuvre attribuée au Salernitain Archimattheus ${ }^{20}$. Surtout, les relations sont de plus en plus souvent codifiées par le recours à l'acte notarié qui vient conclure l'accord entre un professionnel et son client. Ces «pactes de guérison », comme les définit Gianna Pomata ${ }^{21}$, font leur apparition en Occident dans les pays de droit écrit ${ }^{22}$, et visent à définir clairement ce que les deux parties sont en droit d'attendre de leur accord: d'un côté la guérison - qu'il reste encore à définir car la notion même de santé n'est pas une donnée univoque selon qu'elle consiste en un état parfait ou dans la simple amélioration de la partie du corps affectée par la maladie-, de l'autre la juste rétribution. Mais là encore, les dénonciations de ces contrats devant la justice témoignent de ces conceptions souvent divergentes entre praticiens et malades à propos de ce qu'il faut entendre par santé, ou encore illustrent les réticences du malade à suivre le traitement et l'incompétence du médecin.

7 Il est certain cependant que la pratique médicale est un métier à haut risque où la réputation du médecin est engagée, à partir du moment où il se décide à soigner son patient. Danielle Jacquart montre toutefois que si, dans l'Antiquité, plusieurs traités hippocratiques déniaient à la médecine le soin de s'occuper des cas incurables, le Moyen Âge place, lui, la responsabilité du praticien, non dans le résultat, mais dans la capacité à déterminer par son pronostic et son diagnostic les maladies mortelles. La tâche était cependant loin d'être simple en l'absence de concept de ce genre dans les ouvrages médicaux. Aussi, à l'exception de l'expérience des fièvres pestilentielles qui facilitait l'établissement d'un pronostic fatal, le médecin avait-il plutôt tendance à se montrer prudent et réservé dès qu'il s'agissait d'établir un tel pronostic.

8 La prudence est aussi de mise dans sa fonction principale qui est de donner des conseils $^{23}$. Les traités de déontologie qu'étudie Chiara Crisciani visent à réguler les relations que doit établir le praticien, non seulement avec son patient, mais aussi avec ses confrères (médecins, chirurgiens, renommés ou débutants) comme lui consultés, in absentia ou in presentia, par le malade. S'y dessine l'éthique en formation d'une profession encore hétérogène, où l'on cherche aussi bien la vérité et la concorde entre praticiens qui sont utiles au malade, qu'à définir un certain nombre de règles déontologiques.

9 Si les conditions de l'exercice médical font l'objet d'une réflexion de la part des médecins, les autorités urbaines ou les patients eux-mêmes s'efforcent aussi de leur côté de définir le rapport au praticien. À travers l'exemple de la ville catalane de Manresa, Michael McVaugh témoigne du développement d'une sorte de médecine de prévention, en pleine seconde moitié du XIV siècle. L'embauche par un groupe d'habitants de la cité d'un médecin appointé à l'année, le recrutement d'un chirurgien communal ou encore le témoignage du livre de recettes d'un apothicaire qui fournit le nom de ses clients dessinent les contours d'un accès assez large des populations urbaines à la profession médicale et d'une « démocratisation » de ces pratiques.

10 Les trois dernières communications abordent les marges plus ou moins acceptées et revendiquées des compétences médicales. En faisant figurer dans leurs ouvrages de chirurgie des considérations consacrées à l'ornatus et à la decoratio, des auteurs français du XIV $^{e}$ siècle ne s'inscrivent pas seulement dans une tradition arabe; Laurence 
Moulinier montre qu'ils répondent aussi à une demande sociale, qui n'est pas au demeurant uniquement féminine, et qu'ils tentent de délimiter les contours de leur domaine, au moment où les physici s'efforcent de contrôler l'ensemble des pratiques médicales.

Mis à rude épreuve par l'épidémie de peste de 1348 et par ses nombreuses résurgences endémiques, les médecins, poussés par les autorités publiques à fournir des réponses thérapeutiques et prophylactiques, ont pu être tentés, comme le souligne Nicolas WeillParot, de recourir à des explications et à des soins magico-astrologiques; mais cités dans le cadre strict de l'explication scolastique, ces usages médicaux de l'astrologie et ces pratiques empiriques sont demeurés prisonniers des filets d'une pensée rationnelle.

Si astrologie et médecine ont toujours entretenu des rapports complexes, Joseph Ziegler montre qu'à partir du XIII ${ }^{\mathrm{e}}$ siècle les auteurs de physiognomonie font un usage de plus en plus poussé des explications médicales, et notamment de la théorie humorale qui permet de définir la complexion du corps et des organes. Tandis que les médecins se contentent encore d'intégrer ponctuellement la physiognomonie à leurs œuvres médicales, notamment lorsqu'il est question de complexion, des auteurs de la fin $\mathrm{du}$ Moyen Âge fournissent dans leur analyse physiognomonique des portraits proches d'un bilan médical.

\section{NOTES}

* Ces communications, proposées lors d'une table ronde organisée à Lyon les 13 et 14 mars 2003 à l'École normale supérieure-Lettres et Sciences Humaines, comprenaient également une intervention d'Agostino Paravicini Bagliani, qui n'a malheureusement pas pu nous en donner de version écrite. Nous souhaiterions, avec Laurence MoulinierBrogi, exprimer ici notre gratitude aux intervenants pour la qualité de leur participation, et nos remerciements à l'EA 2720 « Transmission et mutations des savoirs antiques » de l'École Pratique des Hautes Études (Paris), au défunt Centre d'Histoire Urbaine, au conseil scientifique de l'ENS-LSH et à Laurent Delacroix.

1. J. Shatzmiller, Jews, Medicine, and Medieval Society, Berkeley-Los Angeles-Londres, 1994, p. 2-8 ; M. McVAUGH, Medicine before the Plague. Practitioners and their Patients in the Crown of Aragon, 1285-1345, Cambridge, 1993.

2. Cf. par exemple l'acte de fondation en 1181 d'une école de médecine en Montpellier sur décision de Guilhem VIII, seigneur de la ville, acte dans lequel ladite création est justifiée au nom du bien public et pour le bienfait de toute la communauté (éd. M. Fournier, Les statuts et privilèges des universités françaises, t. 2, Paris, 1891, p. 3).

3. Sur Montpellier, par exemple, voir L. Moulinier-Brogi, «L'originalité de l'école de médecine de Montpellier ", dans La Medicina nel Medioevo. La « schola Salernitana » e le altre, III Giornate della Scuola Medica Salernitana (Salerne, $1^{\mathrm{er}}$ juin 2002), A. Leone et G. Sangermano éd., Salerne, 2003, p. 101-126 et les actes à paraître du colloque de Montpellier (17-19 mai 2001), L'université de Montpellier et son rayonnement. XIII ${ }^{e}-X V^{e}$ siècles, D. Jacquart et D. Le Blévec dir. 
4. Voir pour la France et l'Italie, D. Jacquart, Le milieu médical en France du XII ${ }^{e} a u$ $X V^{e}$ siècle, Genève, 1981 ; Ead., La médecine médiévale dans le cadre parisien (XIV ${ }^{e}-\mathrm{XV}^{e}$ siècles), Paris, 1998 (Penser la médecine) ; M. McVaugh, op. cit. ; L. Moulinier-Brogi, « Les médecins dans le Centre-Ouest au Moyen Âge », dans Scrivere il Medioevo. Lo spazio, la santità, il cibo. Un libro dedicato ad Odile Redon, B. Laurioux et L. Moulinier-Brogi dir., Rome, 2001, p. 405-429 (I libri di Viella, 28) ; I. Naso, Medici e strutture sanitarie nella società tardo-medievale. Il Piemonte dei secoli XIV e XV, Milan, 1982 ; M. Nicoud, « La médecine à Milan à la fin du Moyen Âge : les composantes d'un milieu professionnel », dans Mires, physiciens, barbiers et charlatans. Les marges de la médecine de l'Antiquité aux débuts de l'époque moderne (Université de Reims, 24 janvier 2003), F. Collard et E. Samama dir., sous presse ; K. Park, Doctors and Medicine in early Renaissance Florence, Princeton, 1985 ; A. Pasi, « Medici e chirurghi toscani alle soglie della rivoluzione scientifica », Nuova Rivista Storica, t. 74, 1990, p. 537-578.

5. M. GREEN, « Documenting Medieval Women's Medical Practice », dans Practical Medicine from Salerno to the Black Death, L. GARCÍA-BALLESTER, R. FRENCH, J. ARRIZABALAGA et A. Cunningham éd., Cambridge, 1994, p. 322-352 ; EAD., Women's Healthcare in the Medieval West : Texts and Contexts, Aldershot, 2000.

6. Cf. V. NutTon, « Continuity or Rediscovery? The City Physician in Classical Antiquity and Medieval Italy ", dans The Town and State Physician in Europe, from the Middle Ages to the Enlightenment, A. W. Russel éd., Wolfenbüttel, 1981, p. 9-46 (Wolfenbütteler Forschungen, 17), rééd. ID., From Democedes to Harvey, Londres, 1988 (Variorum Reprints).

7. Sur la pratique médicale en milieu essentiellement rural et hérétique, voir P. BILLER, " Medicine and Heresy », dans Religion and Medicine in the Middle Ages, éd. P. BILLER et J. ZIEGLER, York, 2001, p. 155-174 (York Studies in Medieval Theology, III).

8. Par exemple, J. ArrizabalagA, « Facing the Black Death : Perception and Reaction of University Medical Practitioners ", dans Practical Medicine..., op. cit., p. 237-288; ou pour un aspect plus institutionnel, C. M. CIPOLLA, Health and Medical Profession in the Renaissance, Cambridge, 1976 ; ID., Contro un nemico invisibile. Epidemie e strutture sanitarie nell'Italia del Rinascimento, Bologne, 1986 ; J. HENDERSON, « The Black Death in Florence : Medical and Communal Responses ", dans Death in Towns, S. BASSETT éd., Leicester, 1992, p. 136-150. Plus récemment, Body and City : History of Urban Public Health, S. PowER et H. SHEARD éd., Londres, 2000.

9. J. Agrimi et C. Crisciani, «L'assistance dans la civilisation chrétienne médiévale », dans Histoire de la pensée médicale en Occident, vol. 1 : Antiquité et Moyen Âge, M. D. Grmek dir., Paris, 1995, p. 151-174 ; M. D. Grmek, « Le médecin au service de l'hôpital médiéval en Europe occidentale ", History and Philosophy of the Life Sciences, t. 4, 1982, p. 25-64 et le volume de A. J. Grieco et L. Sandri dir., Ospedali e città : l'Italia del Centro-Nord, XIII-XVI, Florence, 1997.

10. Voir par exemple les travaux de M. Balint, Le médecin, son malade et la maladie, Paris, 1960 ; C. Herzlich, Santé et maladie. Analyse d'une représentation sociale, Paris, 1969 ; Ead. et J. Pierret, Malades d'hier, malades d'aujourd'hui, Paris, 1984 ou encore F. Laplantine, Anthropologie de la maladie. Étude ethnologique des systèmes de représentations étiologiques et thérapeutiques dans la société occidentale contemporaine, Paris, 1986.

11. D. Gourévitch, Le triangle hippocratique dans le monde gréco-romain. Le malade, sa maladie et son médecin, Rome, 1984 (BEFAR, 251). 
12. Médecine et morale dans l'Antiquité, dans Entretiens sur l'Antiquité classique, H. Flashar et J. Jouanna éd., t. XLIII, Vandœuvres-Genève, 1997.

13. A. Paravicini Bagliani, Medicina e Scienze della Natura alla Corte dei Papi del Duecento, Spolète, 1991.

14. M. McVaugh, op. cit., notamment le premier chapitre.

15. M. Nicoud, «Expérience de la maladie et échange épistolaire : les derniers moments de Bianca Maria Visconti (mai-octobre 1468) ", Mélanges de l'École française de Rome. Moyen Âge, t. 112, 2000, p. 311-458.

16. G. Pomata, La promessa di guarigione. Malati e curatori in antico regime. Bologna XVI-XVIII, Rome-Bari, 1994. Voir également la contribution de C. Crisciani dans le présent volume. 17. Cf. M. Nicoud, «Les médecins à la cour de Francesco Sforza ou comment gouverner le Prince ", dans Actes du colloque en hommage à Jean-Louis Flandrin (Université de ParisVIII, 27-29 novembre 2003), à paraître.

18. P. Gil Sotres, «Les régimes de santé », dans Histoire de la pensée médicale..., op. cit., p. 257-281 ; Id., « Els Regimina sanitatis », dans Regimen sanitatis ad regem Aragonum.

Arnaldi de Villanova Opera medica omnia, éd. L. García-Ballester et M. McVaugh, vol. X.1, Barcelone, 1996, p. 17-110.

19. Voir les diverses communications proposées lors de la table ronde sur Mires, physiciens, barbiers..., op. cit.

20. Archimattheus, De instructione medici, dans Collectio Salernitana, éd. S. DE RENZI, 5 vol., Naples, 1852-59, vol. 2, p. 74-75, et vol. 5, p. 333-334 et 348-349.

21. G. Pomata, op. cit.

22. Voir entre autres Razo, Cahiers du Centre d'Études médiévales de Nice, t. 4, 1984, Le corps souffrant : maladies et médications, dossier : " Guérir au Moyen Âge : médecine et société au xiv ${ }^{\mathrm{e}}$ et $\mathrm{xv}^{\mathrm{e}}$ siècle », p. 109-144 (H. Bresc, " Documents siciliens »; N. Coulet, « Documents aixois »; L. Stouff, « Documents arlésiens », etc.) ; cf. aussi J. Shatzmiller, Médecine et justice en Provence médiévale. Documents de Manosque, 1262-1348, Aix-enProvence, 1989.

23. Sur cette littérature, voir J. Agrimi et C. Crisciani, Les «Consilia » médicaux, Turnhout, 1993 (Typologie des sources du Moyen Âge occidental, fasc. 69).

\section{AUTEUR}

\section{MARILYN NICOUD}

École normale supérieure-Lettres et Sciences Humaines, 15 parvis René Descartes, 69366 Lyon Cedex 07 